75 years of Agricultural University - Plovdiv

JUBILEE SCIENTIFIC INTERNATIONAL

CONFERENCE Plovdiv 26-28 November 2020
PERSPECTIVES ON AGRICULTURAL SCIENCE AND INNOVATIONS FOR SUSTAINABLE FOOD SYSTEMS

\title{
DOI: 10.22620/agrisci.2021.30.002 \\ OVERVIEW OF THE MACHINE VISION METHODS AT AGROROBOTS
}

\author{
Vera Stefanova, Georgi Komitov* \\ Agricultural University - Plovdiv \\ *E-mail: gkomitov@abv.bg
}

\begin{abstract}
The idea of "seeing" machines developed violently after the entry of computer technology and industry 4.0 into mass production. The first task of the machine vision is the processing of images and their adjacent equipment, and its systems then include devices with digital inputs/outputs. These inputs/outputs are designed to control some kind of movement or operation of equipment, such as manipulators or automatic lines. The modern machine vision systems are a modern direction of technology that contains elements of computer technology, optics, mechanical systems and industrial automation. With their help, modern agrorobots perform orderly tasks such as visual identification, visual control, obstacle and position determination.

This article presents an overview of certain applications of different machine vision systems suitable for use in agrorobots. It is known that they are autonomously powered and work in poor climatic conditions. In other cases, the machine vision in agrorobots must necessarily work in random oscillations and variable focal length, as well as with a wide assortment of objects for research. The article discusses the capabilities of different types of cameras and related software, the application of QR and laser technology for agrorobots.
\end{abstract}

Key words: mashine vision, agrorobots, industry 4.0, visual control.

\section{INTRODUCTION}

Attempts to construct "seeable" machines date back to many years ago, but it was only with the development of computer technology that their mass incursion into the field of manufacturing became a reality. It is because of this that the machine vision is still used in the context of the computer vision. However, the computer vision is oriented towards image processing, and the idea of the machine vision is much wider in scope. Together with the processing of images and their associated equipment, devices with digital inputs/outputs intended to control production equipment such as different handles or flow lines shall also be included in the wider scope of the machine vision systems (Vernon, 1991; Jain, 1995, Zohdy, 2019).

The modern machine vision systems are a modern direction of technology that contains elements of computer technology, optics, mechanical systems and industrial automation. The most common application of the machine vision is the quality control of finished or inproduction products in a wide variety of activities, such as the production of integrated circuits, electronic products, cars, food, pharmaceuticals and much more. The modern machine vision systems exclude the subjective human factor, assessing the quality of finished elements using digital smart cameras and image processing software (Cognex, 2016; Inzhenering, 2008). 
75 years of Agricultural University - Plovdiv JUBILEE SCIENTIFIC INTERNATIONAL CONFERENCE Plovdiv 26-28 November 2020
PERSPECTIVES ON AGRICULTURAL SCIENCE AND INNOVATIONS FOR SUSTAINABLE FOOD SYSTEMS
Although some machine vision algorithms have been developed to mimic human visual perception, a significant proportion of existing recognition methods have been developed on the basis of the concept of image processing. It is known that the Latin word perceptio, which means perception, in the field of biology and medicine, is the property of man to perceive the surrounding world. The higher level of perception is called apperception and includes elements of perception or, to put it another way, apperception is the ability to gain new knowledge in relation to knowledge gained (experience) (Inzhenering, 2008).

The modern machine vision systems typically support image processing capabilities, while the computer-based image recognition systems are usually designed to perform single, repetitive tasks. Despite the significant expansion of their functionality and capabilities, there are still no computer or machine vision systems that can combine the possibilities of human vision in the sense of imaging, flexibility in terms of changes in illumination (and thus the subsequent reduction of image clarity), change in the constituent elements of the image, etc. (Hosny, 2017).

The modern smart digital farming is impossible without the application of the machine vision methods. With them, the robot orients itself as it moves, detects diseases and enemies in plants, and recognizes its working inventory. Thanks to the algorithms set, it can make an adequate decision about the application of a particular type of technology in the cultivation of agricultural production with a view to increasing yields.

\section{MATERIALS AND METHODS}

This article provides an overview of the types of CCD and CMOS video sensors, as shown by the main differences between them. Guidance has been given on the location of machine vision elements applied to agrorobots, with an emphasis on the illumination of the capture object.

\section{RESULTS AND DISCUSSION}

The machine vision systems include a complex of elements (Fig. 1). The main element in this complex structure is the camera. It could be one or more. Cameras can be black and white and color, analog or digital. They must be equipped with appropriate optics for this purpose in order to be able to perceive images from what they capture in a dynamic environment. Furthermore, these systems have and maintain a specialized camera interface. With its help, the images are digitized. Its other name is "frame catcher". The function of this catcher is to turn analog video signals into a digital protocol (Cognix, 2016; Inzhenering, 2008; Dechow, 2020).

Any modern machine vision system is ineffective if it cannot transmit the results of what it captures in a more ground-based form. This system always relies on input/output hardware, such as digital inputs/outputs or communication connections (network connection, RS-232, etc.) (Cognix, 2016; Inzhenering, 2008; Vernon, 1991).

Intelligent machine vision systems also include lenses to focus the desired field of image on the image sensor, light filters and suitable, very often specialized light sources - LED emitters, fluorescent or halogen lamps, etc. A synchronizing sensor can also be added to this complex to detect the object for inspection (very often it is an optical or magnetic sensor). His idea is to switch between receiving the image and processing it (Cognix, 2016; Inzhenering, 2008; Vernon, 1991).

Apart from all this, the machine vision systems also require a computer with appropriate software to process the image and make the appropriate decision. In many cases of computer vision, the entire complex listed above requires a serious energy resource and space for 
75 years of Agricultural University - Plovdiv

JUBILEE SCIENTIFIC INTERNATIONAL

CONFERENCE Plovdiv 26-28 November 2020
PERSPECTIVES ON AGRICULTURAL SCIENCE AND INNOVATIONS FOR SUSTAINABLE FOOD SYSTEMS its installation (Dechow, 2020; Mapanga, 2012; Vernon, 1991).

The use of all these elements of machine vision systems, together with appropriate software for image processing, as well as for making adequate decisions and the formation and transmission of tasks to the executive units are the basis of the digitization of agriculture. In addition to the use of intelligent systems and cloud technologies for remote access, the basic principles of industry 4.0 in the agricultural sector are formed.

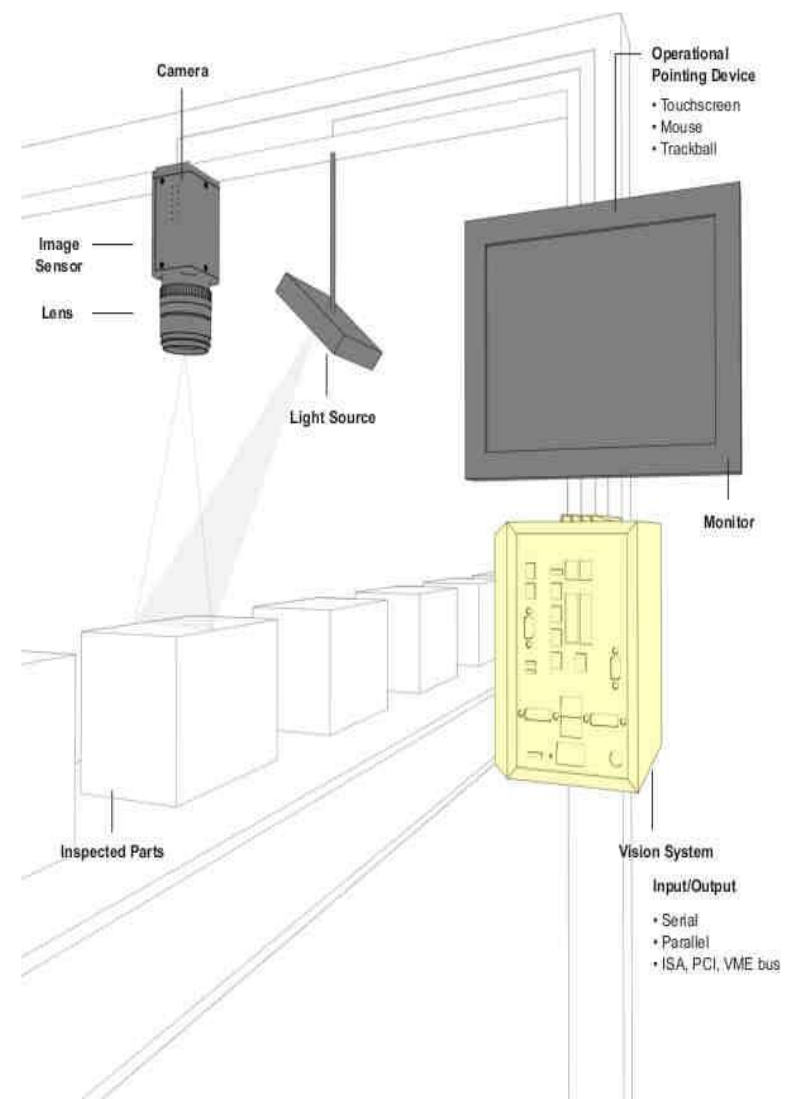

Fig. 1. Elements of the machine vision system

However, in agrorobots, the standard concept is not applicable in practice due to the energy dependence of the robot. All the listed elements are heavy and consume electricity. The heavier the construction becomes or requires a greater consumption the area of the autonomous operation of the robot is reduced. A suitable option is to make a balance in terms of weight/consumption of each element of the system. In addition, it should be noted that most of the elements of the system use standard network voltage for power supply. This is avoided if it is not universal and has a power cord (Mapanga, 2012).

The solution is also in the deconcentration of all elements of the robot frame. There should be left the most important elements such as camera, lenses, filters and lighting. The software is suitable to install on a docking station with constant communication with the robot. Thus, this need for devices for processing and storing the data is removed. The executive outputs are also suitable to be mounted on the robot in order to be able to perform its main operations. It is for these reasons that a specialized machine vision package for agrorobots does not exist, and a number of elements of production systems are brought into practice.

Video cameras generally have tools for recognizing images, brightness, and contrast. Their task is to replace the photoelectric sensors in more complex applications. In the modern video camera solutions, lighting and electronic control are integrated into the device housing. In case of an inspection signal, the sensor compares the current image with the pre-trained and decides yes or no. Typically, these devices have a software option to set up digital outputs. In this way, it is possible to output the solution, after which the signal is processed by a programmable controller and more judgment measures are taken. The possibilities for communication and exchange of data with other systems depend on the manufacturer and the specific model (Cogniex, 2016).

Video cameras are two variants according to the use of different image recognition technology - CCD and CMOS (Fig. 2) (Hosny, 2017). 
75 years of Agricultural University - Plovdiv JUBILEE SCIENTIFIC INTERNATIONAL CONFERENCE Plovdiv 26-28 November 2020
PERSPECTIVES ON AGRICULTURAL SCIENCE AND INNOVATIONS FOR SUSTAINABLE FOOD SYSTEMS
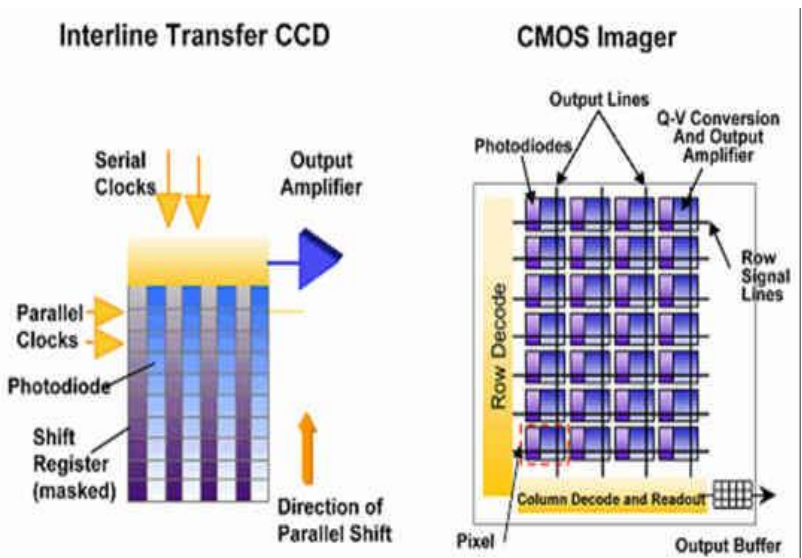

Fig. 2. Difference between CCD and CMOS

All CCD and CMOS image sensors work through the development of the photoelectric effect of converting light into electricity. All CCD and CMOS sensors must perform the following main functions (Holms, 2010; Hosny, 2017):

- generate and collect charge

- measure it and turn into voltage or current

- give an output signal.

Charge coupled device. Literally translated, it means an element with a charger connection or a charge-connected device. This is a specialized analog integrated circuit capable of storing, moving and processing electrical charges. This is a specialized analog integrated chip capable of storing, moving and processing electrical charges. It is made of the element $\mathrm{Si}$ (Fig. 3). (Fellers, 2020).

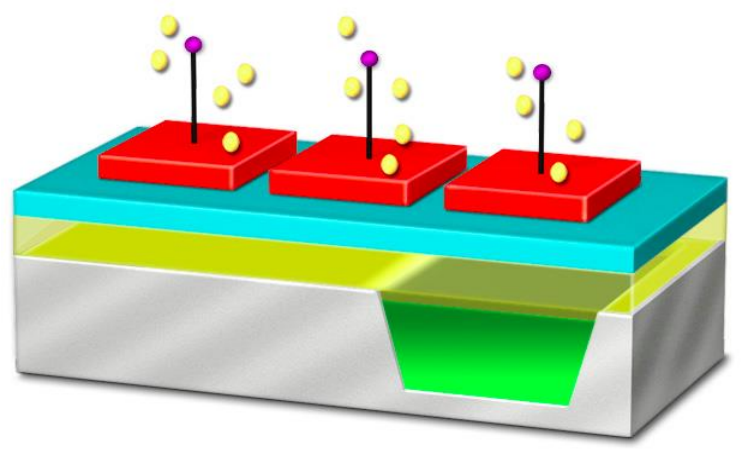

Fig. 3. CCD sensor
The CCD sensors generate voltage according to the amount of incoming light. After exposure, the charges move gradually, electrode by electrode, hence the name of the device "bucket brigade device", until they reach the output amplifier. The charge values of the individual dots (pixels or picture elements) are recorded sequentially, even though they occurred simultaneously. The value of each point (pixel) means the charge proportional to the light intensity. Then, the voltage is removed, depending on the charges or the amount of light. Due to this "analog change controller" in video surveillance, the CCD sensors are mainly used for analog cameras. The CCD sensors are characterized by low noise and high light sensitivity. However, they need an additional image processor (Fellers, 2020).

Most video camera chips record with only half a picture (interlaced), i.e. first the odd lines of the picture, then the even ones, and so on. There are four methods to prevent additional charges from lighting: Full-Frame - (FF), Frame-Transfer - (FT), Interline-Transfer - (IT) and Frame-Interline-Transfer-CCDs (FIT).

The charge-coupled device was invented in 1969 at AT\&T Bell Labs by Willard Boyle and George E. Smith. For the invention of the CCD, Boyle and Smith were awarded the Stuart Ballantin Medal at the Franklin Institute in 1973, the IEEE N. Liebman Prize in 1974, the Charles Stark Prize in 2006, and the Nobel Prize in Physics in 2009.

The Complementary metal oxide semiconductor CMOS. The CMOS sensors, also known as active pixel sensors, collect the charges produced by the incoming light, just like a CCD sensor. However, there is an output amplifier for each pixel, which amplifies the signal and gives it a digital value. Based on the technology, the biggest difference between a CMOS image sensor and a CCD sensor is that every single pixel is read. The CMOS sensors are cheaper to manufacture than other types of CCDs and consume significantly less electricity 
75 years of Agricultural University - Plovdiv JUBILEE SCIENTIFIC INTERNATIONAL CONFERENCE Plovdiv 26-28 November 2020
PERSPECTIVES ON AGRICULTURAL SCIENCE AND INNOVATIONS FOR SUSTAINABLE FOOD SYSTEMS
(Turchetta, 2020).

When a broad wavelength band of visible light is incident on specially doped silicon semiconductor materials, a variable number of electrons are released in proportion to the photon flux density incident on the surface of a photodiode. In effect, the number of electrons produced is a function of the wavelength and the intensity of light striking the semiconductor. Electrons are collected in a potential well until the integration (illumination) period is finished, and then they are either converted into a voltage (CMOS processors) or transferred to a metering register (CCD sensors). The measured voltage or charge (after conversion to a voltage) is then passed through an analog-to-digital converter, which forms a digital electronic representation of the scene imaged by the sensor Fig.4. (Turchetta, 2020).

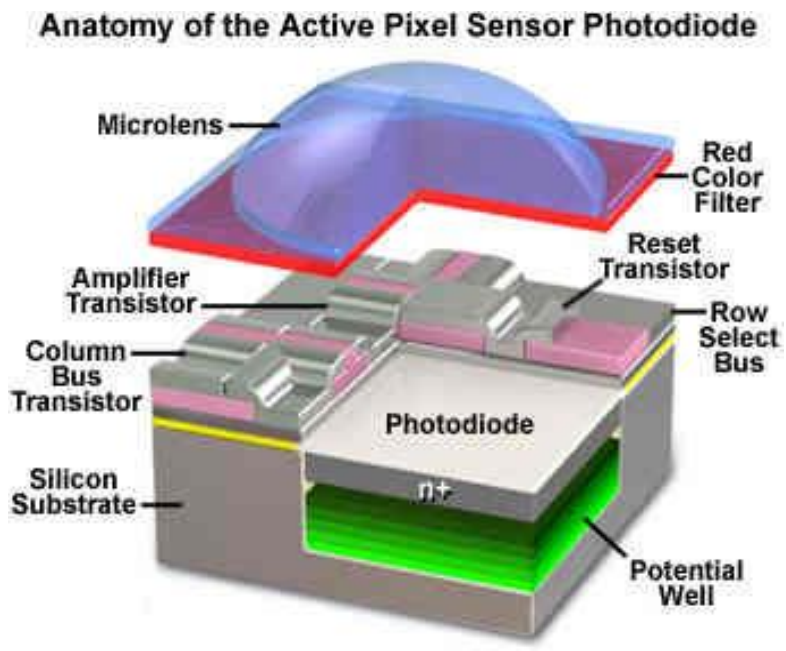

Fig. 4. CMOS sensor

Another key element of the machine vision system is the illumination of the subject. In order to obtain a quality image and to be able to use it for process control, it is necessary that the subject is well lit. With all lighting techniques, it is important to increase the contrast between the subject and the surrounding background. There are several types of ways to illuminate objects depending on the relative position of the camera and the objects to be inspected (Cognix, 2016; Dechow,
2020).

Back lighting enhances an object's outline for applications that need only external or edge measurements. Back lighting helps detect shapes and makes dimensional measurements more reliable.

Axial diffuse lighting couples light into the optical path from the side (coaxially). A semi-transparent mirror illuminated from the side, casts light downwards on the part. The part reflects the light back to the camera through the semi-transparent mirror resulting in a very evenly illuminated and homogeneous looking image.

Structured light is the projection of a light pattern (plane, grid, or a more complex shape) at a known angle onto an object. It can be very useful for providing contrast-independent surface inspections, acquiring dimensional information and calculating volume.

Dark-field illumination - directional lighting more easily reveals surface defects and includes dark-field and bright-field illumination. Dark-field illumination is generally preferred for low-contrast applications. In dark-field illumination, the specular light is reflected away from the camera, and the diffused light from the surface texture and elevation changes are reflected into the camera.

Bright-field illumination is ideal for the high-contrast applications. However, the highly directional light sources such as the highpressure sodium and quartz halogen may produce sharp shadows and generally do not provide consistent illumination throughout the entire field of the view. Consequently, hot-spots and specular reflections on shiny or reflective surfaces may require a more diffused light source to provide even illumination in the brightfield.

Diffused dome lighting gives the most uniform illumination of features of interest, and can mask irregularities that are not of interest and may be confusing to the scene. 
75 years of Agricultural University - Plovdiv JUBILEE SCIENTIFIC INTERNATIONAL CONFERENCE Plovdiv 26-28 November 2020
PERSPECTIVES ON AGRICULTURAL SCIENCE AND INNOVATIONS FOR SUSTAINABLE FOOD SYSTEMS
This is the difference between the standard shooting of plants in detail on the soil. Depending on the moisture content and compaction of the soil light absorption is different. Plants also change the world's photosensitivity, the absorption of light depending on its stage of development. If there are disturbances in the proper development of plants, this will also affect the absorption of light. The presences of moisture, dust on the leaves and detergent residues also have a negative effect on the brightness of the object. The presence of air currents constantly moves the position of the leaves of the plant. All these different background conditions at any given time form a variable complex of quantities and make it impossible to use a standard technique for illuminating the object. This requires the frequent use of two and sometimes three variants of lighting techniques. Suitable variants are diffuse and structured light, as shown in Fig. 5. (Cognix, 2016; Dechow, 2020).

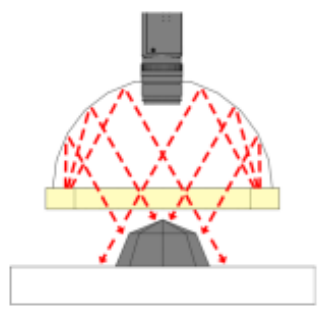

a)

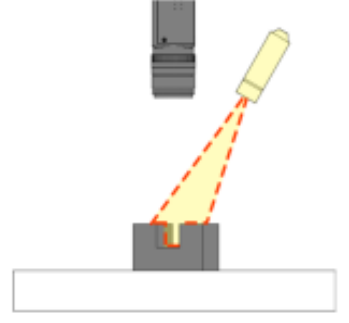

b)
Fig. 5. Light technology a-diffused dome lighting, b-structured light

It is possible to mount a laser and a $\mathrm{QR}$ reader to the system, but their role is only to support or orient the main camera when to start shooting or where the robot is located. They still do not make decisions to perform certain operations.

\section{CONCLUSIONS}

The elements of the machine vision system are considered in this article. Guidelines are given for the application in agricultural robots. The different image recognition technologies are discusses as well.

The main types of technologies for lighting the sites are pointed out, and recommendations for their application in agricultural robots are given.

\section{ACKNOWLEGMENT}

The authors are grateful to the Center for Research, Technology Transfer and Intellectual Property Protection at the Agricultural University - Plovdiv for the subcontract on internal project 02-20 "Power unit for agricultural robot".

\section{REFERENCES}

Cognex (2016). Introduction of machine vision. Dechow, D. (2020). The Fundamentals of Machine Vision. Intelligent Robotics/Machine Vision FANUC America Corporation. AIA-CVP. Acsses on octomber 2020 ww.visiononline.org/userAssets/aiaUpl oads/file/T1-The-Fundamentals-ofMachine-Vision.pdf

Fellers, T., Davidson, M. (2020). Introduction to Charge-Coupled Devices (CCDs). Acsses on octomber 2020 https://www.microscopyu.com/digitalimaging/introduction-to-chargecoupled-devices-ccds

Holms, A., Quach, A. (2010). Complementary Metal-Oxide Semiconductor Sensors.

Hosny, M., Omari, M., (2017). CMOS Image Sensors. DOI: 10.13140/RG.2.2.29423.84644

Inzhenering Revyu (2008). Mashinno zrenie. Inzhenering Revyu, vol. 1. p. 66-76.

Jain, R., Kasturi, R., Schunck, B., 1995. Mashine vision. McGraw-Hill, Inc.

Mapanga, K., Kumar, V. (2012). Machine Vision for intelligent Semi-Autonomous Transport (MV-iSAT). International 
75 years of Agricultural University - Plovdiv JUBILEE SCIENTIFIC INTERNATIONAL CONFERENCE Plovdiv 26-28 November 2020
PERSPECTIVES ON AGRICULTURAL SCIENCE AND INNOVATIONS FOR SUSTAINABLE FOOD SYSTEMS

Symposium on Robotics and Intelligent Sensors 2012. Procedia Engineering 41. $395-404$

Turchetta, R., Davidson, M. (2020). Introduction to CMOS Image Sensors. Acsses on octomber 2020 https://micro.magnet.fsu.edu/primer/dig italimaging/cmosimagesensors.html

Vernon, D. (1991). Machine vision. Automated Visual Inspection and Robot Vision. Prentice Hall.

Zohdy, B., Mahmood, M., Darwish, N., Hefny, H. (2019). Chapter 8. Machine Vision Application on Science and Industry: Machine vision Trends. Computational Intelligence and Robotics (ACIR) Book Series. IGI Global. p. 233-254. 